Supporting Information

\title{
Electrospray Ionization Inlet Tandem Mass Spectrometry (ESII-MS/MS): A Hyphenated Method for the Sensitive Determination of Chemicals in Animal Tissues and Body Fluids
}

\author{
Shubhashis Chakrabarty, Weilin L. Shelver, and David J. Smith* \\ USDA-Agricultural Research Service, Edward T. Schafer Agricultural Research Center, \\ Biosciences Research Laboratory, 1616 Albrecht Blvd, Fargo, ND 58102, USA.
}

*Corresponding author: David J. Smith

Email: David.j.smith@,usda.gov

Phone: (701) 239-1238

Fax: (701) 239-1430 


\section{Table of Contents}

Page \#

Figure S1: ESII ion chromatograms of $\mathrm{m} / \mathrm{z} 185$ obtained from zilpaterol in different matrices

Table S1: Concentration of flunixin and 5-OH flunixin in cow urine collected on

S4 dosing day 3 .

Table S2: $\quad$ Concentration of 5-OH flunixin in cow urine collected on dosing day $1 . \quad$ S5 
(a)
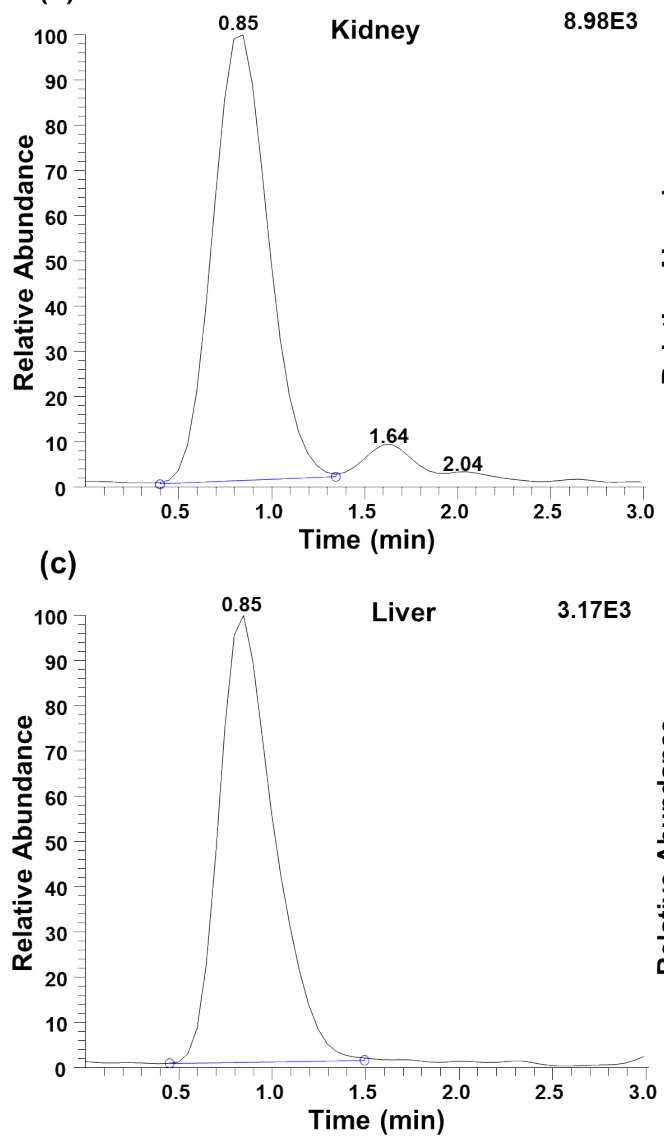

(b)

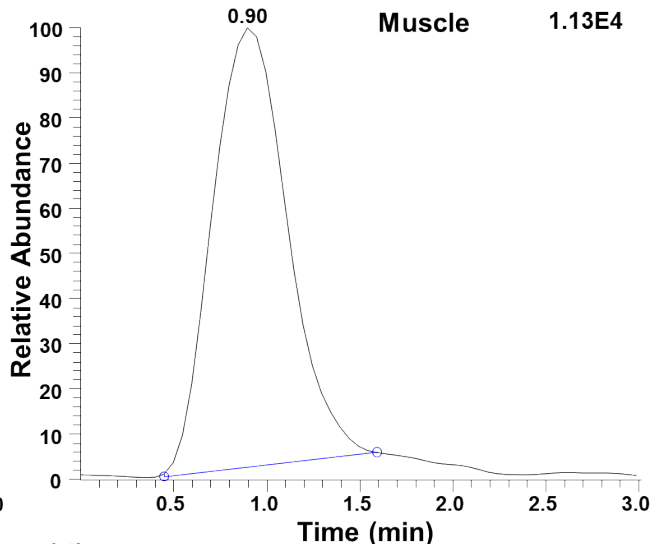

(d)

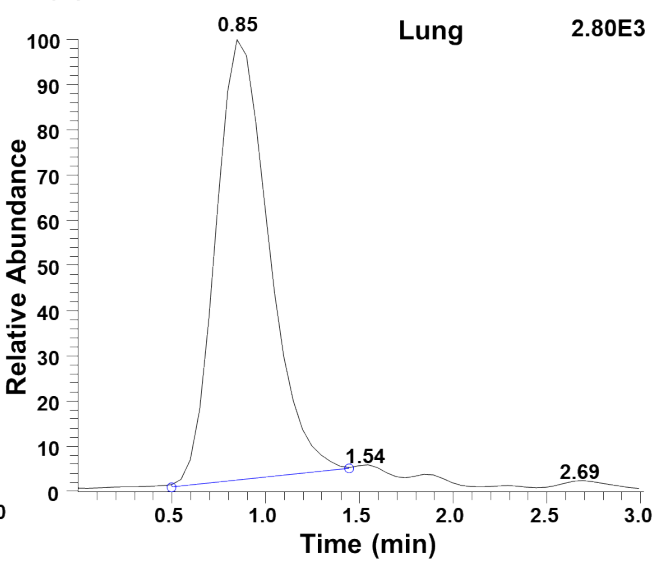

Figure S1. ESII-MS ion chromatograms of $\mathrm{m} / \mathrm{z} 185$ obtained from $4 \mathrm{ng} / \mathrm{g}$ zilpaterol fortified in different matrices a) kidney; b) muscle; c) liver and d) lung. 
Table S1. Concentration of flunixin and 5-hydroxy flunixin in cow urine collected on dosing day 3 at 24, 48, 72 and $96 \mathrm{~h}$ from cows 2, 3, 4 and 5 and analyzed using ESII-MS/MS and LCMS/MS.

\begin{tabular}{ccccc}
\hline \multirow{2}{*}{ Samples } & \multicolumn{2}{c}{ Flunixin $(\mathbf{n g} / \mathbf{m L})$} & \multicolumn{2}{c}{ 5-Hydroxy Flunixin $\mathbf{( n g} / \mathbf{m L})$} \\
\cline { 2 - 5 } & ESII-MS/MS & LC-MS/MS & ESII-MS/MS & LC-MS/MS \\
\hline Cow-2 D3T24 & 3040 & 3620 & 390 & 340 \\
Cow-2 D3T48 & 960 & 1060 & 200 & 75 \\
Cow-2 D3T72 & 370 & 345 & - & - \\
Cow-2 D3T96 & 230 & 150 & - & - \\
Cow-3 D3T24 & 1050 & 980 & 300 & 185 \\
Cow-3 D3T48 & - & - & - & - \\
Cow-3 D3T72 & - & - & - & - \\
Cow-3 D3T96 & - & - & - & - \\
Cow-4 D3T24 & 2080 & 3570 & 490 & 390 \\
Cow-4 D3T48 & 360 & 345 & 180 & 30 \\
Cow-4 D3T72 & 220 & 85 & - & - \\
Cow-4 D3T96 & - & - & - & - \\
Cow-5 D3T24 & 1190 & 1760 & 360 & 250 \\
Cow-5 D3T48 & - & - & - & - \\
Cow-5 D3T72 & - & - & - & - \\
Cow-5 D3T96 & - & - & - & - \\
\hline
\end{tabular}


Table S2. Concentration of 5-hydroxy flunixin in cow urine collected on dosing day 1 at $2 \mathrm{~h}$ from 17 cows and analyzed using ESII-MS/MS and LC-MS/MS. In ESII-MS/MS the standard curve was prepared from single injection of standard solutions.

\begin{tabular}{ccc}
\hline & \multicolumn{2}{c}{ 5-Hydroxy Flunixin $(\mathbf{n g} / \mathbf{m L})$} \\
\cline { 2 - 3 } Samples & \multicolumn{2}{c}{} \\
\cline { 2 - 3 } & ESII-MS/MS & LC-MS/MS \\
\hline Cow1 D1T2 & 6436 & 7000 \\
Cow2 D1T2 & 15918 & 19045 \\
Cow3 D1T2 & 1934 & 2165 \\
Cow4 D1T2 & 3109 & 3145 \\
Cow5 D1T2 & 7977 & 8205 \\
Cow6 D1T2 & 982 & 1090 \\
Cow7 D1T2 & 1326 & 1465 \\
Cow8 D1T2 & 3486 & 3785 \\
Cow9 D1T2 & 2435 & 2040 \\
Cow10 D1T2 & 1065 & 1060 \\
Cow11 D1T2 & 4945 & 5065 \\
Cow12 D1T2 & 10677 & 10615 \\
Cow16 D1T2 & 5526 & 5100 \\
Cow17 D1T2 & 6488 & 6130 \\
Cow18 D1T2 & 1513 & 1350 \\
Cow19 D1T2 & 25113 & 24970 \\
Cow20 D1T2 & 12449 & 11585 \\
\hline
\end{tabular}

